\title{
A Hands-On Lab for Macro Malware Detection using Machine Learning on Virtual Machines
}

\author{
Dan C. Lo, Ruth Bearden, \\ Deepa Muralidhar, Hossain Shahriar \\ Dept. of Computer Science \\ Kennesaw State University \\ Marietta, GA, U.S.A \\ \{dlo2, rbeard13, dmurali1, \\ hshahria\}@kennesaw.edu
}

\author{
Wei Chen \\ Dept. of Computer \\ Science \\ Tennessee State University \\ Nashville, TN, U.S.A. \\ wchen@tnstate.edu
}

\author{
Pascal Paschos \\ Dept. of Computer \\ Science \\ Chicago State University \\ Chicago, IL, U.S.A. \\ ppaschos@csu.edu
}

\author{
Chung Ng \\ Dept. of Computer \\ Science \\ Morehouse College \\ Atlanta, GA, U.S.A. \\ Chung.Ng@morehouse.edu
}

\begin{abstract}
We developed a hands-on lab for students to learn macro malware detection using decision trees on an open sourced data analytics software, HPCC, running on a virtual machine. The labware along with required software and documents are freely available at our project website. Our design is low cost, customizable and can be easily replicated to other institutions. Our experience shows that students have difficulties in configuring software environment for the lab. The virtual machine approach allows pre-configurations for datasets and required software installations and setting to facilitate students' learning and instructors' teaching. Since students are working on malware, the virtual machine provides an isolated network environment for testing without affecting normal operating network. This lab design can also be integrated into online courses by simply downloading and installing the customized virtual machine.
\end{abstract}

\section{CCS CONCEPTS}

- Security and privacy Malware and its mitigation

- Applied computing Computer-managed instruction

- Applied computing Digital libraries and archives

KEYWORDS: Computer Science Education; Information Security; Cybersecurity; Virtual Machines; Virtualization

\section{INTRODUCTION}

Hands-On experiments are essential when educating cybersecurity experts. However, it is difficult for cybersecurity education to keep pace with rapidly changing computer security issues to mimic real-world scenarios in a contained environment. Virtual Machine Introspection (VMI) is a technique that has been deeply investigated in intrusion detection, malware analysis, and memory forensics. With the success of VMI, it is possible to teach hands-on labs on kernel level security that allows students to learn (1) stack-based buffer over-flow, (2) direct kernel object manipulation (DKOM), and (3) kernel integrity checker.

Permission to make digital or hard copies of part or all of this work for personal or classroom use is granted without fee provided that copies are not made or distributed for profit or commercial advantage and that copies bear this notice and the full citation on the first page. Copyrights for third-party components of this work must be honored. For all other uses, contact the Owner/Author.

SIGCSE'20, March 11-14, 2020, Portland, OR, USA.

(c) 2020 Copyright is held by the owner/author(s).

ACM ISBN 978-1-4503-6793-6/20/03. https://doi.org/10.1145/3328778.3372576
Our learning platform, a laptop with a virtual box, provides an environment for security testing. It will leverage the advantages of the VM platform to enable learning anywhere anytime. All the slides, tutorials, and demonstrations will be designed to be included in a VM. All the security exercises can be performed using the VM along with necessary software. All materials are accessible from our dedicated repository. This helps in reducing the adoption curve, cost, and broadening the cybersecurity education.

Macro malware is a piece of executable code embedded in nonexecutable documents such as Microsoft (MS) Office files. A recent incidence of a ransomware variant exploits Microsoft Office Macro, attached in an email as a document with ".docm" extension, to execute evil code and ransomware has resulted in a loss of $\$ 8$ billion and listed top 5 cybersecurity threads in 2018 . Our method of classifying MS Office files containing macros as malicious or benign relies on decision tree machine learning algorithms, feature selection, and term frequency-inverse document frequency (TFIDF) where p-code (Microsoft's proprietary intermediate languages) opcode n-grams (translated Visual Basic for Applications (VBA) macro code) compose the file features. Our dataset in this lab includes a sample set of 40 malicious and 118 benign MS Office files containing macros. The preliminary study demonstrates the effectiveness of our proposed approach to teaching students macro malware detection using machine learning on HPCC systems. An instance of HPCC ECL Watch is running on a Linux Ubuntu (64-bit) in a VirtualBox.

We have built a VM that contains all necessary pre-configured software and ready for students to learn. This lab design can also be integrated into online courses by simply downloading and installing the customized virtual machine. Learning materials include pre-lab, in-lab, and post-lab learning modules are freely available at our project website:

https://sites.google.com/site/iasoncs/home/malware-detectionusing-machine-learning.

\section{ACKNOWLEDGMENTS}

The work is partially supported by the U.S. National Science Foundation under awards: 1723586, 1723578, 1636995, 1623724. Any opinions, findings, and conclusions or recommendations expressed in this material are those of the authors and do not necessarily reflect the views of the National Science Foundation. 